\section{Prognosefaktoren bei angeborener Zwerchfellhernie}

Schwere Lungenhypoplasie und persistierende pulmonale Hypertension bestimmen wesentlich das Überleben von Neugeborenen mit angeborener Zwerchfellhernie. Ziel einer internationalen Studie war es, den Stellenwert des Oxygenierungsindex als frühen Prognoseparameter zu überprüfen.

$D^{i}$ konnatale Zwerchfellhernie (CDH, „congenital diaphragmatic hernia“) ist eine komplexe Erkrankung mit einer Inzidenz von 0,45 auf 1.000 Lebendgeborene. Meist findet sich der Zwerchfelldefekt linksseitig. 50\% der Fälle weisen einen auffälligen Karyotyp oder weitere anatomische Fehlbildungen wie Herzfehler oder Lippen-KieferGaumen-Spalten auf. Bekanntlich haben Kinder, die erst nach den ersten 10 Lebensminuten respiratorische Probleme entwickeln, eine gute Prognose. Sehr selten findet sich zufällig im Röntgenthorax eine $\mathrm{CDH}$ auch bei älteren Kindern (Abb. 1).

In eine retrospektive Studie konnten 235 Neugeborene mit CDH aufgenommen werden. Die Patienten verteilten sich auf vier Zentren: 29 in Helsinki, 64 in Madrid, 113 in London und 29 in Graz. In Helsinki und Graz steht die Möglichkeit zur neonatalen extrakorporalen Membranoxygenierung (ECMO) und in London für sehr schwere Fälle die Möglichkeit zur fetalen endoluminalen trachealen Okklusion (FETO) zur Verfügung. Bei nur einem Patienten wurde

ECMO und bei 66 FETO eingesetzt. Signifikante Prognoseparameter für ein Überleben an Lebenstag 28 waren ,liverdown“-Position ( $\mathrm{p}<0,0001)$, ,lung-tohead"-Ratio $>1(\mathrm{p}=0,003)$ und primärer Komplettverschluss ohne „patch“ ( $\mathrm{p}$ $=0,02)$. Die Gesamtüberlebensrate betrug 67,6\%. Als bedeutungsvollster Prognoseparameter erwies sich der „beste Oxygenierungsindex am 1. Lebenstag“. day 1: a reliable marker for outcome and survival in infants with congenital diaphragmatic hernia. Eur J Pediatr Surg 2015;25:3-8

\section{Kommentar}

Im Rahmen der Erstversorgung eines Neugeborenen mit CDH ist eine Maskenbeatmung kontraindiziert, da die Mediastinalverlagerung durch die Luftfüllung von Magen und Darm die Lungenkompression verstärken. Bezüglich Beatmungsstrategie besteht derzeit ein Trend zu weniger ECMO und mehr inhalativem Stickstoffmonoxid. Überraschend ist trotz ECMO-Verfügbarkeit die sehr niedrige ECMO-Rate in
Ruttenstock E et al. Best oxygenation index on

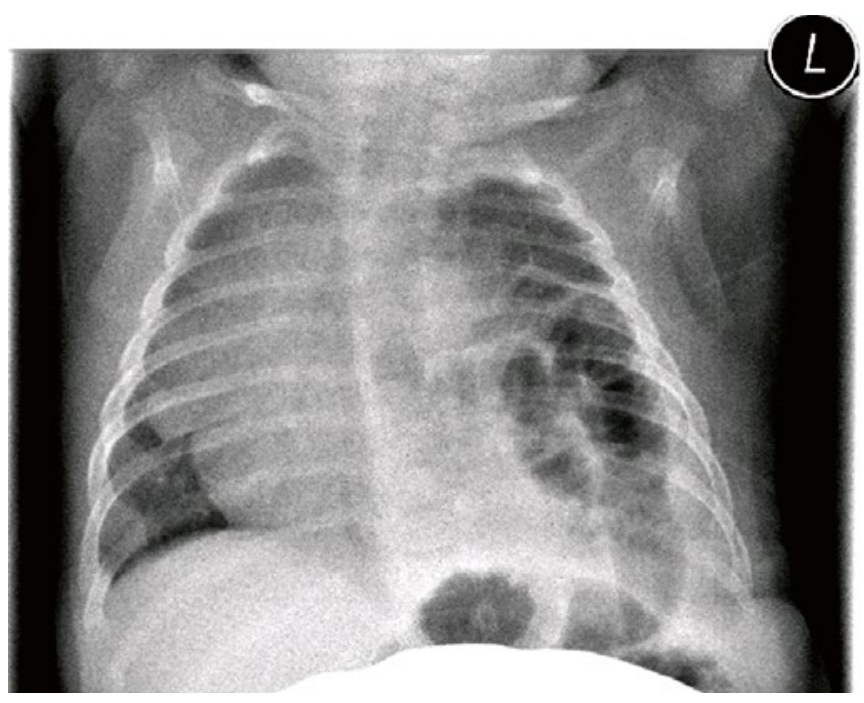

Tab. 1: Indikationen zur ECMO [nach Extracorporal Life Support Registry Report 2014]

- Oxygenierungsindex $>40>4$ Stunden

- Oxygenierungsindex $>20>24$ Stunden ohne Zeichen der Besserung trotz maximaler medikamentöser Therapie oder bei persistierenden Episoden der Dekompensation

- schweres hypoxisches Versagen mit akuter Dekompensation $\left(\mathrm{PaO}_{2}<40\right.$ $\mathrm{mmHg}$ ) ohne Reaktion auf Intervention

_ zunehmendes respiratorisches Versagen und/oder pulmonale Hypertension mit Nachweis einer rechtsventrikulären Dysfunktion oder anhaltender Katecholaminbedarf

der Studienpopulation. ECMO sollte nur in Zentren mit entsprechender Fallzahl und Erfahrung für Patienten wie beispielsweise mit großem Zwerchfelldefekt und kritischer Beatmungssituation durchgeführt werden. Zur frühen Kalkulation, Intensivierung der Beatmungs- und Kreislauftherapie und zeitlich optimaler Verlegungsplanung hat die Erhebung des "besten Oxygenierungsindex am ersten Lebenstag" beziehungsweise die Dynamik der Entwicklung dieser Werte eine praktisch große Bedeutung. Definition des Oxygenierungsindex: Mittlerer Atemwegsdruck $\left(\mathrm{cmH}_{2} \mathrm{O}\right) \times \mathrm{FiO}_{2}(\%) / \mathrm{PaO}_{2}(\mathrm{mmHg})$.

Tab. 1 listet die Indikationen zur ECMO nach dem Extracorporal Life Support Registry Report 2014. Jedes ECMO-Zentrum verfügt meist über eigene Kriterien. Allerdings bestehen auch zahlreiche Kontraindikationen. Eine aktuelle Originalarbeit aus dem Dr. von Haunerschen Kinderspital / Perinatalzentrum der LMU München [Förster KM et al. Z Geburtsh Neonatol 2015;219:274-80] konnte positive Resultate vorweisen: Nach einem vorbereitenden und weiter begleitenden tierexperimentellen ECMO-Trainingsprogramm an einem spezialisierten Großklinikum mit umfassenden Möglichkeiten der Therapie schwer kranker Neugeborener konnten im nationalen und internationalen Vergleich gute Behandlungsergebnisse wenn auch bei noch kleiner Fallzahl - erzielt werden. Von den insgesamt 13 ECMO-Patienten zwischen 2008-2014 hatten fünf eine $\mathrm{CDH}$, wovon vier überlebten.

Dr. Thomas Hoppen 